\title{
Foreign Exchange Derivative Pricing with Stochastic Correlation
}

\author{
Topilista Nabirye ${ }^{1}$, Philip Ngare ${ }^{2}$, Joseph Mungatu ${ }^{3}$ \\ ${ }^{1}$ Pan African University, Institute of Basic Science, Technology and Innovation, JKUAT, Nairobi, Kenya \\ ${ }^{2}$ School of Mathematics, University of Nairobi, Nairobi, Kenya \\ ${ }^{3}$ Department of Statistics, School of Mathematics, Jomo Kenyatta University of Agriculture and Technology, Nairobi, Kenya \\ Email: topistanabirye3@gmail.com
}

How to cite this paper: Nabirye, T., Ngare, P. and Mungatu, J. (2016) Foreign Exchange Derivative Pricing with Stochastic Correlation. Journal of Mathematical Finance, 6, 887-899.

http://dx.doi.org/10.4236/jmf.2016.65059

Received: October 7, 2016

Accepted: November 20, 2016

Published: November 23, 2016

Copyright (C) 2016 by authors and Scientific Research Publishing Inc. This work is licensed under the Creative

Commons Attribution International

License (CC BY 4.0).

http://creativecommons.org/licenses/by/4.0/

c) (i) Open Access

\begin{abstract}
Financial markets are known to be far from deterministic but stochastic and hence time dependent correlation tends to suit the markets. We price for European Options by using three dimensional assets under stochastic correlation. The pricing equations under constant correlation and stochastic correlation are derived numerically by using finite difference method called the Crank Nicolson method. We compare the pricing equations when the correlation is stochastic and constant by using real data from emerging financial markets, that is, exchange rates data for Kenya as the domestic currency and South Africa as the foreign currency. Pricing equation for the European option with stochastic correlation performed better than that with constant correlation.
\end{abstract}

\section{Keywords}

Foreign Exchange, European Option, Stochastic Correlation and Option Pricing

\section{Introduction}

The long history of option pricing began in 1900 when the French mathematician Louis Bachelier deduced an option pricing formula based on the assumption that stock prices followed a Brownian motion with zero drift [1]. Since 1900, many theories and models have been developed to cater for the behaviour of financial markets. In 1965, Samuelson [2] proposed a popular model for the behaviour of asset prices. In 1973, Black and Scholes [3] provided an equation called the Black-Scholes equation to price derivatives on a single asset in the Black-Scholes model which modified Samuelson model. In 1985, Cox, Ingersoll and Ross [4] extended the Black-Scholes equation to the generalised Black-Scholes equation to price derivatives on multiple assets. Most models used in the pricing of multidimensional derivatives consider constant correlation among their components but empirical facts suggest that correlation varies over time. Therefore ignoring 
changes in the correlation may introduce significant misleading in the pricing. The stochastic correlations have been proposed by different researchers, see for example [5] [6] [7] [8], among others.

In [7], closed-form approximation as well as a measure of the error for the price of two dimensional derivatives under the assumptions of stochastic correlation and constant volatility was provided. They provided a simulations-free approximation to the price of Spread Options and Quantos Options under non-constant correlation. They provided a framework for pricing two-dimensional derivatives under time dependent correlation together with a bound for the error and without the need for time-con- suming numerical methods.

A reasonable and appropriate time-dependent correlation function is built so that one can reasonably choose additional parameters to increase the fitting quality on the one hand but also add an economic concept on the other hand [9]. Thus many problems of finance and economics can be treated under dynamic correlation which is much more realistic than with a constant correlation to model real world phenomena.

In [10], instead of assuming a constant correlation, they developed a strategy for pricing the Quanto option under dynamic correlation in a closed formula, including the calibration to market data. They also compared the pricing and hedging strategy with and without dynamic correlation and studied the effect of dynamic correlation on the option pricing and hedging. [6] dealt with the stochastic modelling of correlation in finance where they illustrated the evidence that the correlation was hardly a deterministic quantity with the analysis of correlation between daily returns time series of S and P Index and Euro/USD exchange rates. They also determined a transition density function of the stochastic correlation processes in closed form and computed the price of a quantity adjusting option (Quanto).

However, all the literatures we have come across on stochastic correlation dealt with either one dimensional derivative or two dimensional derivatives. In this study, we intend to price European Options by using three dimensional assets under stochastic correlation. The study is divided into four sections, that is, pricing with constant correlation, pricing when the correlation is stochastic, numerical results and conclusion.

\section{Pricing Equations for European Options under Constant Correlation}

Consider the European call $C$ to be a function of $S(t) A^{*}(t, T), A(t, T), X, T$ where $S(t)$ is the spot domestic currency price of a unit of foreign exchange at time $t$, $A^{*}(t, T)$ is the foreign currency price of a pure discount bond which pays one unit of foreign exchange at time $t+T, A(t, T)$ is the domestic currency price of a pure discount bond which pays one unit of domestic currency at time $t+T, X$ is the domestic currency exercise price of an option on foreign currency, $t$ is the initial time and $T$ is the expiration time.

The following assumptions are to be considered.

$C$ has the general functional form 


$$
C=C\left[S(t) A^{*}(t, T), A(t, T), X, T\right]
$$

subjected to the boundary conditions

$$
\begin{aligned}
& C[S(t+T), X, 0]=\max [0, S(t+T)-X] \\
& C[0, A(t, T), X, T]=0
\end{aligned}
$$

where Equation (1) is the terminal value of the call option, which has to be greater than zero or the strike value and Equation (2) means that when the spot exchange value is zero, then option to be bought has a zero value.

The second assumption has to do with the dynamics of $S, A^{*}$, and $A$. Let $\mathrm{d} W_{1}, \mathrm{~d} W_{2}$, $\mathrm{d} W_{3}$ denote standardized Wiener processes with unit instantaneous variances and correlation matrix

$$
\left(\begin{array}{ccc}
1 & \rho_{S A^{*}} & \rho_{S A} \\
\rho_{S A^{*}} & 1 & \rho_{A^{*} A} \\
\rho_{S A} & \rho_{A^{*} A} & 1
\end{array}\right) \mathrm{d} t
$$

where $\rho_{w}=\rho_{w}(t, T)$ can be a known function of time $(t)$ and the time to maturity of the bond (T). Assume $S, A^{*}, A$ follow the Geometric Brownian Motions

$$
\begin{aligned}
& \frac{\mathrm{d} S}{S}=\mu_{S}(t) \mathrm{d} t+\sigma_{S}(t) \mathrm{d} W_{1} \\
& \frac{\mathrm{d} A^{*}}{A^{*}}=\mu_{A^{*}}(t, T) \mathrm{d} t+\sigma_{A^{*}}(t, T) \mathrm{d} W_{2} \\
& \frac{\mathrm{d} A}{A}=\mu_{A}(t, T) \mathrm{d} t+\sigma_{A}(t, T) \mathrm{d} W_{3}
\end{aligned}
$$

Basing on the assumption above, we can define new variables $\mathrm{d} H, \mathrm{~d} W_{4}$, and using Ito's product rule,

$$
\frac{\mathrm{d} H}{H}=\frac{\mathrm{d}\left(S A^{*}\right)}{S A^{*}}=\left(\mu_{S}+\mu_{A^{*}}+\rho_{S A^{*}} \sigma_{S} \sigma_{A^{*}}\right) \mathrm{d} t+\sigma_{S} \mathrm{~d} W_{1}+\sigma_{A^{*}} \mathrm{~d} W_{2}
$$

with a correlation coefficient between them $\rho_{S A^{*}} \mathrm{~d} t=\mathrm{d} W_{1} \mathrm{~d} W_{2}$

$$
\frac{\mathrm{d} H}{H}=\mu_{H}(t, T) \mathrm{d} t+\sigma_{H}(t, T) \mathrm{d} W_{4}
$$

and write the correlation matrix of $\mathrm{d} W_{4}, \mathrm{~d} W_{3}$ as

$$
\left(\begin{array}{cc}
1 & \rho_{H A} \\
\rho_{H A} & 1
\end{array}\right) \mathrm{d} t
$$

where $\rho_{H A}=\rho_{H A}(t, T)$.

Applying Ito's lemma to the function

$$
C=C\left[S(t) A^{*}(t, T), A(t, T), X, T\right]=C[H(t, T), A(t, T), X, T]
$$

we get the option dynamic as: 


$$
\begin{aligned}
\mathrm{d} C & =\frac{\partial C}{\partial t} \mathrm{~d} t+\frac{\partial C}{\partial H} \mathrm{~d} H+\frac{\partial C}{\partial A} \mathrm{~d} A \\
& +\frac{1}{2}\left(\frac{\partial^{2} C}{\partial H^{2}} H^{2} \sigma_{H}^{2}+2 \frac{\partial^{2} C}{\partial H \partial A} H A \rho_{H A} \sigma_{H} \sigma_{A}+\frac{\partial^{2} C}{\partial A^{2}} A^{2} \sigma_{A}^{2}\right) \mathrm{d} t
\end{aligned}
$$

Let $\theta$ represent elements involving second derivative and $\tau=T-t$ then $\mathrm{d} t=-\mathrm{d} \tau$, so Equation (7) becomes:

$$
\mathrm{d} C=\frac{\partial C}{\partial \tau} \mathrm{d} \tau+\frac{\partial C}{\partial H} \mathrm{~d} H+\frac{\partial C}{\partial A} \mathrm{~d} A-\frac{1}{2} \theta \mathrm{d} \tau
$$

Let $F$ be a portfolio composed of one option, $b$ units of $H$, and $p$ units of $A$, then:

$$
F=C+b H+p A
$$

The dynamics of this portfolio are:

$$
\mathrm{d} F=\mathrm{d} C+b \mathrm{~d} H+p \mathrm{~d} A
$$

Choose $b, p$ such that $b=-\frac{\partial C}{\partial H}, p=-\frac{\partial C}{\partial A}$, then:

$$
\mathrm{d} F=\left(\frac{\partial C}{\partial \tau}-\frac{1}{2} \theta\right) \mathrm{d} \tau
$$

If the portfolio $F$ uses no wealth, then in equilibrium it should yield a zero return.

$$
F=C-\frac{\partial C}{\partial H} H-\frac{\partial C}{\partial A} A
$$

That is, if $F=0$, then $\mathrm{d} F=0$ which implies that

$$
\frac{\partial C}{\partial \tau}=\frac{1}{2} \theta
$$

We look for a function $C(H, A, X, T)$ that solves Equation (9) and is also subjected to the boundary conditions (1-2). According to [11], the solution to the European call is given by:

$$
C(t)=S(t) A^{*}(t, T) N\left(d_{1}\right)-X A(t, T) N\left(d_{2}\right)
$$

where $N(d)$ is the standard normal distribution with mean 0 and variance 1 and

$$
\begin{gathered}
d_{1}=\frac{\ln \left(\frac{S A^{*}}{X A}\right)+\frac{\sigma^{2}}{2} T}{\sigma \sqrt{T}} \\
d_{2}=\frac{\ln \left(\frac{S A^{*}}{X A}\right)-\frac{\sigma^{2}}{2} T}{\sigma \sqrt{T}} \\
\sigma^{2}=\int_{0}^{T} \frac{1}{T}\left[\sigma_{H}^{2}(t+T-u, u)+\sigma_{A}^{2}(t+T-u, u)\right. \\
\left.-2 \rho_{H A}(t+T-u, u) \sigma_{H}(t+T-u, u) \sigma_{A}(t+T-u, u)\right] \mathrm{d} u
\end{gathered}
$$

But we shall solve Equation (9) numerically and compare with that of stochastic correlation. 


\section{Pricing Equations for European Options under Stochastic Correlation}

Consider Equation (6) and (5)

$$
\begin{gathered}
\frac{\mathrm{d} H}{H}=\mu_{H}(t, T) \mathrm{d} t+\sigma_{H}(t, T) \mathrm{d} W_{4} \\
\frac{\mathrm{d} A}{A}=\mu_{A}(t, T) \mathrm{d} t+\sigma_{A}(t, T) \mathrm{d} W_{3}
\end{gathered}
$$

with a correlation coefficient between them

$$
\mathrm{d} W_{3} \mathrm{~d} W_{4}=\rho \mathrm{d} t
$$

where

$$
\mathrm{d} \rho=a(m-\rho) \mathrm{d} t+c \sqrt{1-\rho^{2}} \mathrm{~d} W_{5}
$$

$a(m-\rho)$ is the drift term, $c \sqrt{1-\rho^{2}}$ is the volatility term and the bound for correlation is $-1 \leq \rho \leq 1$. We assume

$$
\begin{aligned}
& \mathrm{d} W_{4} \mathrm{~d} W_{5}=\rho_{1} \mathrm{~d} t \\
& \mathrm{~d} W_{3} \mathrm{~d} W_{5}=\rho_{2} \mathrm{~d} t
\end{aligned}
$$

where $\rho_{1}$ and $\rho_{2}$ are constants.

The correlation matrix become $\left(\begin{array}{ccc}1 & \rho & \rho_{1} \\ \rho & 1 & \rho_{2} \\ \rho_{1} & \rho_{2} & 1\end{array}\right)$ which must be positive definite that is its determinant is zero or positive. Using Ito's Lemma, we obtain a three-dimensional stochastic differential of the differential Equations (5), (6) and (11)

$$
\begin{aligned}
\mathrm{d} C= & \frac{\partial C}{\partial H} \mathrm{~d} H+\frac{\partial C}{\partial A} \mathrm{~d} A+\frac{\partial C}{\partial \rho} \mathrm{d} \rho \\
& +\left(\frac{\partial C}{\partial t}+\frac{1}{2} \frac{\partial^{2} C}{\partial H^{2}} H^{2} \sigma_{H}^{2}+\frac{1}{2} \frac{\partial^{2} C}{\partial A^{2}} A^{2} \sigma_{A}^{2}+\frac{1}{2} \frac{\partial^{2} C}{\partial \rho^{2}} \rho^{2} \sigma_{\rho}^{2}\right. \\
& \left.+\frac{\partial^{2} C}{\partial H \partial A} H A \rho \sigma_{H} \sigma_{A}+\frac{\partial^{2} C}{\partial H \partial \rho} H \rho_{1} \sigma_{H} \sigma_{\rho}+\frac{\partial^{2} C}{\partial A \partial \rho} A \rho_{2} \sigma_{A} \sigma_{\rho}\right) \mathrm{d} t
\end{aligned}
$$

where $\sigma_{\rho}=c \sqrt{1-\rho^{2}}$

We assume that, under the risk-neutral measure $Q, H$ and $A$ are geometric Brownian motions with mean $r$ (the risk-free interest rate) and constant volatilities $\sigma_{H}>0$, $\sigma_{A}>0$, with respect to Brownian motions $W_{4}, W_{3}$ satisfying;

$$
\begin{gathered}
\frac{\mathrm{d} H}{H}=r \mathrm{~d} t+\sigma_{H}(t, T) \mathrm{d} W_{4} \\
\frac{\mathrm{d} A}{A}=r \mathrm{~d} t+\sigma_{A}(t, T) \mathrm{d} W_{3}
\end{gathered}
$$

Substituting Equations (13), (14) and (11) in Equation (12) we get; 


$$
\begin{aligned}
\mathrm{d} C= & {\left[\frac{\partial C}{\partial t}+\frac{\partial C}{\partial H} r H+\frac{\partial C}{\partial A} r A+\frac{\partial C}{\partial \rho} a(m-\rho)+\frac{1}{2} \frac{\partial^{2} C}{\partial H^{2}} H^{2} \sigma_{H}^{2}+\frac{1}{2} \frac{\partial^{2} C}{\partial A^{2}} A^{2} \sigma_{A}^{2}\right.} \\
& \left.+\frac{1}{2} \frac{\partial^{2} C}{\partial \rho^{2}} \rho^{2} \sigma_{\rho}^{2}+\frac{\partial^{2} C}{\partial H \partial A} H A \rho \sigma_{H} \sigma_{A}+\frac{\partial^{2} C}{\partial H \partial \rho} H \rho_{1} \sigma_{H} \sigma_{\rho}+\frac{\partial^{2} C}{\partial A \partial \rho} A \rho_{2} \sigma_{A} \sigma_{\rho}\right] \mathrm{d} t \\
& +\frac{\partial C}{\partial H} \sigma_{H} H \mathrm{~d} W_{4}+\frac{\partial C}{\partial A} \sigma_{A} A \mathrm{~d} W_{3}+\frac{\partial C}{\partial \rho} \sigma_{\rho} \mathrm{d} W_{5}
\end{aligned}
$$

To obtain the price of the option, following the Black-Scholes analysis, we consider two different options, $C_{1}\left(H, A, K_{1}, T_{1}\right)$ and $C_{2}\left(H, A, K_{2}, T_{2}\right)$ on $H, A, \rho$;

We define a portfolio $F$ by

$$
F=C_{1}+\lambda_{1} C_{2}+\lambda_{2} H+\lambda_{3} A
$$

where $\lambda_{1}, \lambda_{2}$ and $\lambda_{3}$ are units

We assume that $F$ is self-financing. It follows that the dynamics of this portfolio are:

$$
\begin{aligned}
\mathrm{d} F= & \mathrm{d} C_{1}+\lambda_{1} \mathrm{~d} C_{2}+\lambda_{2} \mathrm{~d} H+\lambda_{3} \mathrm{~d} A \\
\mathrm{~d} F=[ & \frac{\partial C_{1}}{\partial t}+\frac{\partial C_{1}}{\partial H} r H+\frac{\partial C_{1}}{\partial A} r A+\frac{\partial C_{1}}{\partial \rho} a(m-\rho)+\frac{1}{2} \frac{\partial^{2} C_{1}}{\partial H^{2}} H^{2} \sigma_{H}^{2}+\frac{1}{2} \frac{\partial^{2} C_{1}}{\partial A^{2}} A^{2} \sigma_{A}^{2} \\
& \left.+\frac{1}{2} \frac{\partial^{2} C_{1}}{\partial \rho^{2}} \rho^{2} \sigma_{\rho}^{2}+\frac{\partial^{2} C_{1}}{\partial H \partial A} H A \rho \sigma_{H} \sigma_{A}+\frac{\partial^{2} C_{1}}{\partial H \partial \rho} H \rho_{1} \sigma_{H} \sigma_{\rho}+\frac{\partial^{2} C_{1}}{\partial A \partial \rho} A \rho_{2} \sigma_{A} \sigma_{\rho}\right] \mathrm{d} t \\
& +\lambda_{1}\left[\frac{\partial C_{2}}{\partial t}+\frac{\partial C_{2}}{\partial H} r H+\frac{\partial C_{1}}{\partial A} r A+\frac{\partial C_{1}}{\partial \rho} a(m-\rho)+\frac{1}{2} \frac{\partial^{2} C_{2}}{\partial H^{2}} H^{2} \sigma_{H}^{2}+\frac{1}{2} \frac{\partial^{2} C_{2}}{\partial A^{2}} A^{2} \sigma_{A}^{2}\right. \\
& \left.+\frac{1}{2} \frac{\partial^{2} C_{2}}{\partial \rho^{2}} \rho^{2} \sigma_{\rho}^{2}+\frac{\partial^{2} C_{2}}{\partial H \partial A} H A \rho \sigma_{H} \sigma_{A}+\frac{\partial^{2} C_{2}}{\partial H \partial \rho} H \rho_{1} \sigma_{H} \sigma_{\rho}+\frac{\partial^{2} C_{2}}{\partial A \partial \rho} A \rho_{2} \sigma_{A} \sigma_{\rho}\right] \mathrm{d} t \\
& +\lambda_{2} r H \mathrm{~d} t+\lambda_{3} r A \mathrm{~d} t+\left[\frac{\partial C_{1}}{\partial H} \sigma_{H} H+\frac{\partial C_{2}}{\partial H} \lambda_{1} \sigma_{H} H+\lambda_{2} \sigma_{H} H\right] \mathrm{d} W_{4} \\
& +\left[\frac{\partial C_{1}}{\partial A} \sigma_{A} A+\frac{\partial C_{2}}{\partial A} \lambda_{1} \sigma_{A} A++\lambda_{3} \sigma_{A} A\right] \mathrm{d} W_{3}+\left[\frac{\partial C_{1}}{\partial \rho}+\lambda_{1} \frac{\partial C_{2}}{\partial \rho}\right]\left[\sigma_{\rho}\right] \mathrm{d} W_{5}
\end{aligned}
$$

For the portfolio $F$ to be risk neutral, the factors in front of $\mathrm{d} W_{3}, \mathrm{~d} W_{4}$ and $\mathrm{d} W_{5}$ need to be zero. This can be achieved by letting $\lambda_{1}, \lambda_{2}$ and $\lambda_{3}$ be:

$$
\begin{aligned}
& \lambda_{1}=-\frac{\partial C_{1} / \partial \rho}{\partial C_{2} / \partial \rho} \\
& \lambda_{2}=\frac{\partial C_{1} / \partial \rho}{\partial C_{2} / \partial \rho} \frac{\partial C_{2}}{\partial H}-\frac{\partial C_{1}}{\partial H} \\
& \lambda_{3}=\frac{\partial C_{1} / \partial \rho}{\partial C_{2} / \partial \rho} \frac{\partial C_{2}}{\partial H}-\frac{\partial C_{1}}{\partial H}
\end{aligned}
$$

The choices of $\lambda_{1}, \lambda_{2}, \lambda_{3}$ above make the portfolio risk neutral, so by absence of arbitrage it must hold that $\mathrm{d} F=r F \mathrm{~d} t$. This means that 


$$
\begin{aligned}
& \Phi_{1} \mathrm{~d} t-\frac{\partial C_{1} / \partial \rho}{\partial C_{2} / \partial \rho} \Phi_{2} \mathrm{~d} t+\left(\frac{\partial C_{1} / \partial \rho}{\partial C_{2} / \partial \rho} \frac{\partial C_{2}}{\partial H}-\frac{\partial C_{1}}{\partial H}\right) r H \mathrm{~d} t+\left(\frac{\partial C_{1} / \partial \rho}{\partial C_{2} / \partial \rho} \frac{\partial C_{2}}{\partial H}-\frac{\partial C_{1}}{\partial H}\right) r A \mathrm{~d} t \\
& =r\left[C_{1}-C_{2}\left(\frac{\partial C_{1} / \partial \rho}{\partial C_{2} / \partial \rho}\right)+\left(\frac{\partial C_{1} / \partial \rho}{\partial C_{2} / \partial \rho} \frac{\partial C_{2}}{\partial H}-\frac{\partial C_{1}}{\partial H}\right) H+\left(\frac{\partial C_{1} / \partial \rho}{\partial C_{2} / \partial \rho} \frac{\partial C_{2}}{\partial H}-\frac{\partial C_{1}}{\partial H}\right) A\right] \mathrm{d} t
\end{aligned}
$$

where $\Phi_{1}, \Phi_{2}$ refer to the $\mathrm{d} t$ terms of $\mathrm{d} C_{1}$ respectively $\mathrm{d} C_{2}$.

Simplifying Equation (16), we get:

$$
\frac{\Phi_{1}-r C_{1}}{\partial C_{1} / \partial \rho} \mathrm{d} t=\frac{\Phi_{2}-r C_{2}}{\partial C_{2} / \partial \rho} \mathrm{d} t
$$

Clearly the left-hand side of Equation (17) does not depend on $C_{2}$, and the right- hand side does not depend on $C_{1}$, so both sides of the equation do not depend on $C_{1}$ and $C_{2}$, so are equal to a function $y(H, A, \rho, t)=\rho y$, which can be considered a premium for correlation risk. This tells us that the price process of a derivative $C$ is a solution of the PDE

$$
\Phi-r C-y \rho \frac{\partial C}{\partial \rho}=0
$$

where $\Phi$ is the $\mathrm{d} t$ term of $\mathrm{d} C$. Writing Equation (18) out fully gives us;

$$
\begin{aligned}
& \frac{\partial C}{\partial t}+\frac{\partial C}{\partial H} r H+\frac{\partial C}{\partial A} r A+\frac{1}{2} \frac{\partial^{2} C}{\partial H^{2}} H^{2} \sigma_{H}^{2}+\frac{1}{2} \frac{\partial^{2} C}{\partial A^{2}} A^{2} \sigma_{A}^{2}+\frac{1}{2} \frac{\partial^{2} C}{\partial \rho^{2}} \rho^{2} \sigma_{\rho}^{2} \\
& +\frac{\partial^{2} C}{\partial H \partial A} H A \rho \sigma_{H} \sigma_{A}+\frac{\partial^{2} C}{\partial H \partial \rho} H \rho_{1} \sigma_{H} \sigma_{\rho}+\frac{\partial^{2} C}{\partial A \partial \rho} A \rho_{2} \sigma_{A} \sigma_{\rho}-r C \\
& +[a(m-\rho)-y \rho] \frac{\partial C}{\partial \rho}=0
\end{aligned}
$$

Since we are dealing with zero coupon bonds, Equation (19) becomes;

$$
\begin{aligned}
& \frac{\partial C}{\partial t}+\frac{1}{2} \frac{\partial^{2} C}{\partial H^{2}} H^{2} \sigma_{H}^{2}+\frac{1}{2} \frac{\partial^{2} C}{\partial A^{2}} A^{2} \sigma_{A}^{2}+\frac{1}{2} \frac{\partial^{2} C}{\partial \rho^{2}} \rho^{2} \sigma_{\rho}^{2} \\
& +\frac{\partial^{2} C}{\partial H \partial A} H A \rho \sigma_{H} \sigma_{A}+\frac{\partial^{2} C}{\partial H \partial \rho} H \rho_{1} \sigma_{H} \sigma_{\rho}+\frac{\partial^{2} C}{\partial A \partial \rho} A \rho_{2} \sigma_{A} \sigma_{\rho} \\
& +[a(m-\rho)-y \rho] \frac{\partial C}{\partial \rho}=0
\end{aligned}
$$

Equation (20) is valid for any option on foreign exchange with underlying measured in foreign currency but paid in domestic one. Since we only need A to hedge, a solution independent of the exchange rate could be figured out. Rewriting the solution $C(H, A, t)=V(H, t)$ and letting $\tau=T-t$, we get:

$$
\frac{\partial V}{\partial \tau}=\frac{1}{2} \frac{\partial^{2} V}{\partial H^{2}} H^{2} \sigma_{H}^{2}+\frac{1}{2} \frac{\partial^{2} V}{\partial \rho^{2}} \rho^{2} \sigma_{\rho}^{2}+\frac{\partial^{2} V}{\partial H \partial \rho} H \rho_{1} \sigma_{H} \sigma_{\rho}+[a(m-\rho)-y \rho] \frac{\partial V}{\partial \rho}
$$

The payoff at expiration time $C(H, T)=\bar{A} \operatorname{Max}[H(T)-X, 0]$, where $\bar{A}$ is a fixed exchange rate.

We solve Equation (21) by finite difference methods that are the Crank-Nicolson method to increase the accuracy and stability of the solution. 


\section{Crank-Nicolson Method}

Notice that Equation (21) has three variables and so we employ three indices. Let the time variable be indexed as $i, H$ as $j$ and $\rho$ as $k$ so that our equation is then discretized as:

$$
\begin{aligned}
\frac{V_{j, k}^{i+1}-V_{j, k}^{i}}{\Delta \tau}= & \frac{H^{2} \sigma_{H}^{2}}{2}\left(\frac{1}{2}\left[\frac{V_{j+1, k}^{i+1}-2 V_{j, k}^{i+1}+V_{j-1, k}^{i+1}}{(\Delta H)^{2}}+\frac{V_{j+1, k}^{i}-2 V_{j, k}^{i}+V_{j-1, k}^{i}}{(\Delta H)^{2}}\right]\right) \\
& +\frac{\rho^{2} \sigma_{\rho}^{2}}{2}\left(\frac{1}{2}\left[\frac{V_{j, k+1}^{i+1}-2 V_{j, k}^{i+1}+V_{j, k-1}^{i+1}}{(\Delta \rho)^{2}}+\frac{V_{j, k+1}^{i}-2 V_{j, k}^{i}+V_{j, k-1}^{i}}{(\Delta \rho)^{2}}\right]\right) \\
& +H \rho_{1} \sigma_{H} \sigma_{\rho}\left(\frac{1}{2}\left[\frac{V_{j+1, k}^{i+1}-2 V_{j, k}^{i+1}+V_{j, k-1}^{i+1}}{\Delta H \Delta \rho}+\frac{V_{j+1, k}^{i}-2 V_{j, k}^{i}+V_{j, k-1}^{i}}{\Delta H \Delta \rho}\right]\right) \\
& +[a(m-\rho)-y \rho] \frac{V_{j, k}^{i+1}-V_{j, k}^{i}}{\Delta \rho}
\end{aligned}
$$

Equation (22) can be organized as;

$$
\begin{aligned}
& \frac{V_{j, k}^{i+1}}{\Delta \tau}-\left(\frac{H^{2} \sigma_{H}^{2}}{4(\Delta H)^{2}}\left(V_{j+1, k}^{i+1}-2 V_{j, k}^{i+1}+V_{j-1, k}^{i+1}\right)+\frac{\rho^{2} \sigma_{\rho}^{2}}{4(\Delta \rho)^{2}}\left(V_{j, k+1}^{i+1}-2 V_{j, k}^{i+1}+V_{j, k-1}^{i+1}\right)\right. \\
& \left.+\frac{H \rho_{1} \sigma_{H} \sigma_{\rho}}{2 \Delta H \Delta \rho}\left(V_{j+1, k}^{i+1}-2 V_{j, k}^{i+1}+V_{j, k-1}^{i+1}\right)+\frac{[a(m-\rho)-y]}{\Delta \rho} V_{j, k}^{i+1}\right) \\
& =\frac{V_{j, k}^{i}}{\Delta \tau}+\frac{H^{2} \sigma_{H}^{2}}{4(\Delta H)^{2}}\left(V_{j+1, k}^{i}-2 V_{j, k}^{i}+V_{j-1, k}^{i}\right)+\frac{\rho^{2} \sigma_{\rho}^{2}}{4(\Delta \rho)^{2}}\left(V_{j, k+1}^{i}-2 V_{j, k}^{i}+V_{j, k-1}^{i}\right) \\
& +\frac{H \rho_{1} \sigma_{H} \sigma_{\rho}}{2 \Delta H \Delta \rho}\left(V_{j+1, k}^{i}-2 V_{j, k}^{i}+V_{j, k-1}^{i}\right)+\frac{[a(m-\rho)-\rho y]}{\Delta \rho} V_{j, k}^{i}
\end{aligned}
$$

Now let

$$
W=\Delta \tau \frac{H^{2} \sigma_{H}^{2}}{4(\Delta H)^{2}}, X=\Delta \tau \frac{\rho^{2} \sigma_{\rho}^{2}}{4(\Delta \rho)^{2}}, Y=\Delta \tau \frac{H \rho_{1} \sigma_{H} \sigma_{\rho}}{2 \Delta H \Delta \rho}, Z=\Delta \tau \frac{[a(m-\rho)-\rho y]}{\Delta \rho}
$$

such that we have the equation given by;

$$
\begin{aligned}
& V_{j, k}^{i+1}-\left[W\left(V_{j+1, k}^{i+1}-2 V_{j, k}^{i+1}+V_{j-1, k}^{i+1}\right)+X\left(V_{j, k+1}^{i+1}-2 V_{j, k}^{i+1}+V_{j, k-1}^{i+1}\right)\right. \\
& \left.+Y\left(V_{j+1, k}^{i+1}-2 V_{j, k}^{i+1}+V_{j, k-1}^{i+1}\right)+Z V_{j, k}^{i+1}\right] \\
& =V_{j, k}^{i}+W\left(V_{j+1, k}^{i}-2 V_{j, k}^{i}+V_{j-1, k}^{i}\right)+X\left(V_{j, k+1}^{i}-2 V_{j, k}^{i}+V_{j, k-1}^{i}\right) \\
& +Y\left(V_{j+1, k}^{i}-2 V_{j, k}^{i}+V_{j, k-1}^{i}\right)+Z V_{j, k}^{i}
\end{aligned}
$$

\section{Numerical Results}

Data from the daily closing exchange rates of Kenya and South Africa was used which was got from OANDA

(https://www.oanda.com/solutions-for-business/historical-rates/main.html) starting from 1 January 2010 to 31 December 2015 and in total 1837 observations. MatLab and R softwares were used. Exchange rates for Kenya were considered to be the domestic currency 
and South Africa, the foreign currency. In financial time series there are trends and the trends are nearly impossible to predict and difficult to characterize mathematically. We usually analyze the so-called log-returns, that is, the logged-value of today's value divided by the one of yesterday. Let $p_{t}$ and $p_{t-1}$ denote the closing exchange rate at the current time $(t)$ and previous day $(t-1)$ respectively, log returns or continuously compounded returns at any time are given by:

$$
r_{t}=\log \left(\frac{p_{t}}{p_{t-1}}\right)
$$

Table 1 presents the summary statistics for the daily closing exchange rates returns of kenya and South Africa. These include the mean, standard deviation, Kurtosis and skewness. Kurtosis is significantly greater than three which implies that they are heavily tailed which is characteristic of financial time series data (All series display significant leptokurtic behavior as evidenced by the large kurtosis with respect to the Gaussian distribution). All returns series have an observation of 1836 . They are all left skewed that is the left tail is longer.

Figure 1 shows the evolution of the daily exchange rates that is for Kenya and South Africa respectively. Both series have trends (which imply that the mean is non constant). Generally, the trend of the Kenya exchange rates data exhibits a decline between 2010 and 2011 and between 2013 and 2014. However, the South Africa exchange rates data exhibit an upward trend in 2012. From a visual analysis, the graph reveals that there is a co-movement of the trends in a similar direction either upward or downward within the period under consideration.

Table 1. Descriptive statistics of returns.

\begin{tabular}{ccc}
\hline Statistics & USD.KES & USD.ZAR \\
Maximum & $4.538 e^{-02}$ & 0.0736700 \\
Minimum & $-5.215 e-02$ & -0.1136000 \\
Mean & $4.111 e-05$ & -0.0000063 \\
Standard deviation & 0.004345903 & 0.01012737 \\
Kurtosis & 44.01931 & 13.91015 \\
Skewness & -0.3129154 & -0.1390287 \\
\hline
\end{tabular}



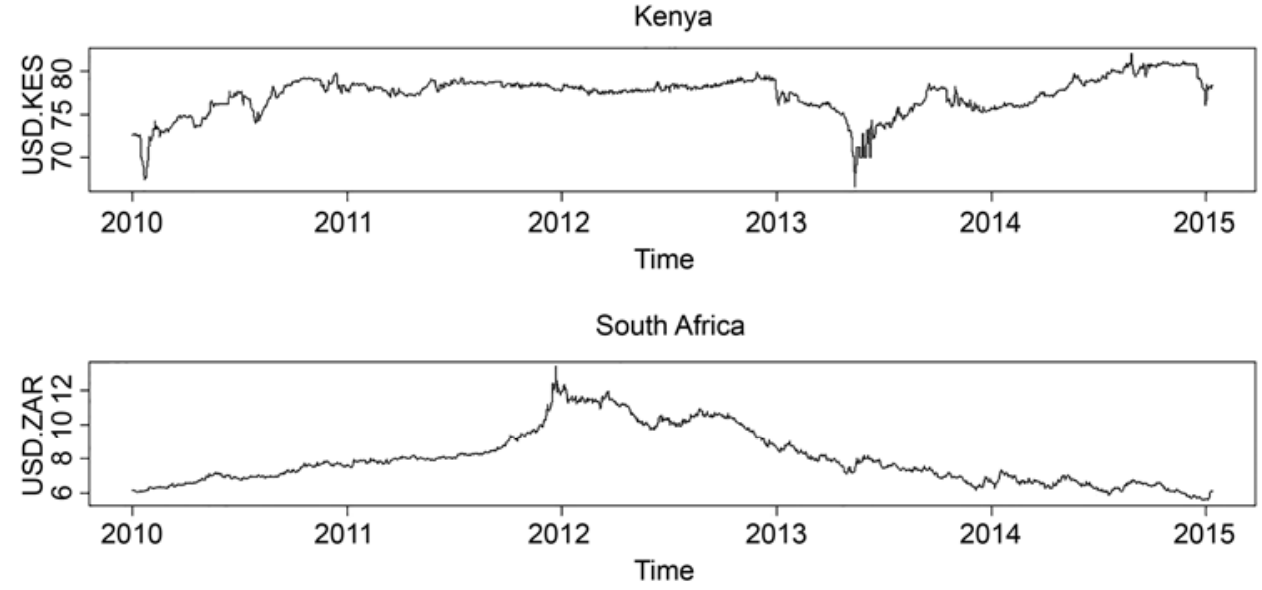

Figure 1. Distribution of the exchange rates.

Daily log returns on exchange rates data are presented in Figure 2 and exhibits no trends. The two graphs reveal the features of financial time series where volatility large clusters and asymmetric are evident.

Figure 3 presents the serial correlation of the returns. It can be seen clearly from Figure 3 that returns exhibits no serial correlation. Thus there is no direct dependency which could be exploited to predict tomorrow's returns based on today or previous days.

The following values of the parameters were used (Table 2):

Data was used to compute some parameter such as $\sigma_{H}, \rho_{H A}$ and $\sigma_{A}$. And parameters a, $m$ and $y$ were just assumed. We were able to assume these parameters after


Figure 2. Distribution of the returns. 
Series USD.KESreturns

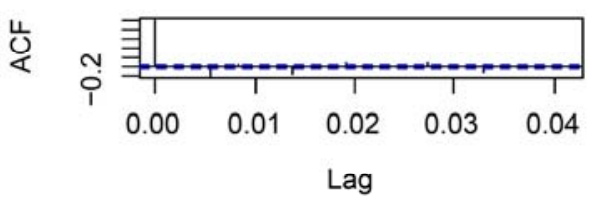

Series USD.ZARreturns

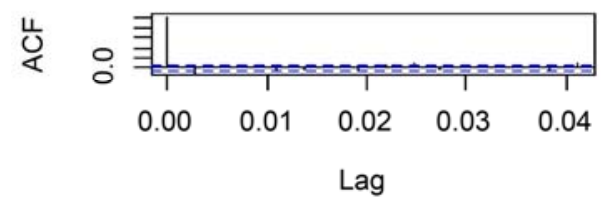

Series USD.KESreturns

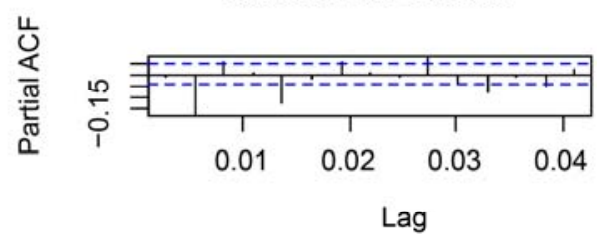

Series USD.ZARreturns

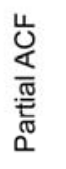

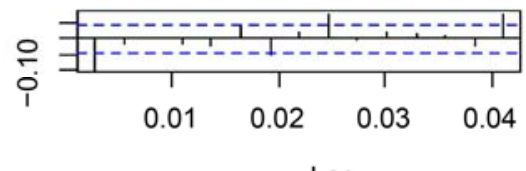

Figure 3. Distribution for serial correlation.

Table 2. Parameters used.

\begin{tabular}{cccccc}
\hline$\sigma_{\text {H }}$ & $\rho_{\text {нА }}$ & $\sigma_{\text {A }}$ & $a$ & $m$ & $y$ \\
\hline 0.01 & -0.0027 & 0.0043 & $\frac{0.001}{1-\rho}$ & 1 & 2 \\
\hline
\end{tabular}

knowing the interval of the parameters of Cox-Ingersoll-Ross(CIR) process since our correlation dynamics is a CIR process.

The parameters above were used to solve Equation (23) and the one for constant correlation. The output is given in the Figures 4-6:

Figure 4 gives the mesh for the prices of the European call when the correlation is constant at maturity time $T=10$.

Figure 5 gives the mesh for the prices of the European call when the correlation is stochastic at maturity time $T=10$.

Figure 6 shows the comparison of European call option prices for stock prices using the constant and stochastic correlation where some parameters are determined from the real data and others assumed. From the Figure 6, it can be seen that the graph for the prices with stochastic correlation performs better since it is close to that of the market prices than the one of constant correlation.

\section{Conclusion}

We price for European Call Options by using three dimensional derivatives under stochastic correlation where other researchers have been using two dimensional derivatives. The pricing formulas for the European call options for constant and stochastic correlation were derived numerically by using the finite difference method called the Crank Nicolson method. Prices for European call for constant and stochastic correlation were compared through using real data from emerging financial markets, that is, the exchange rates data of Kenya and South Africa. Exchange rates for Kenya was considered 


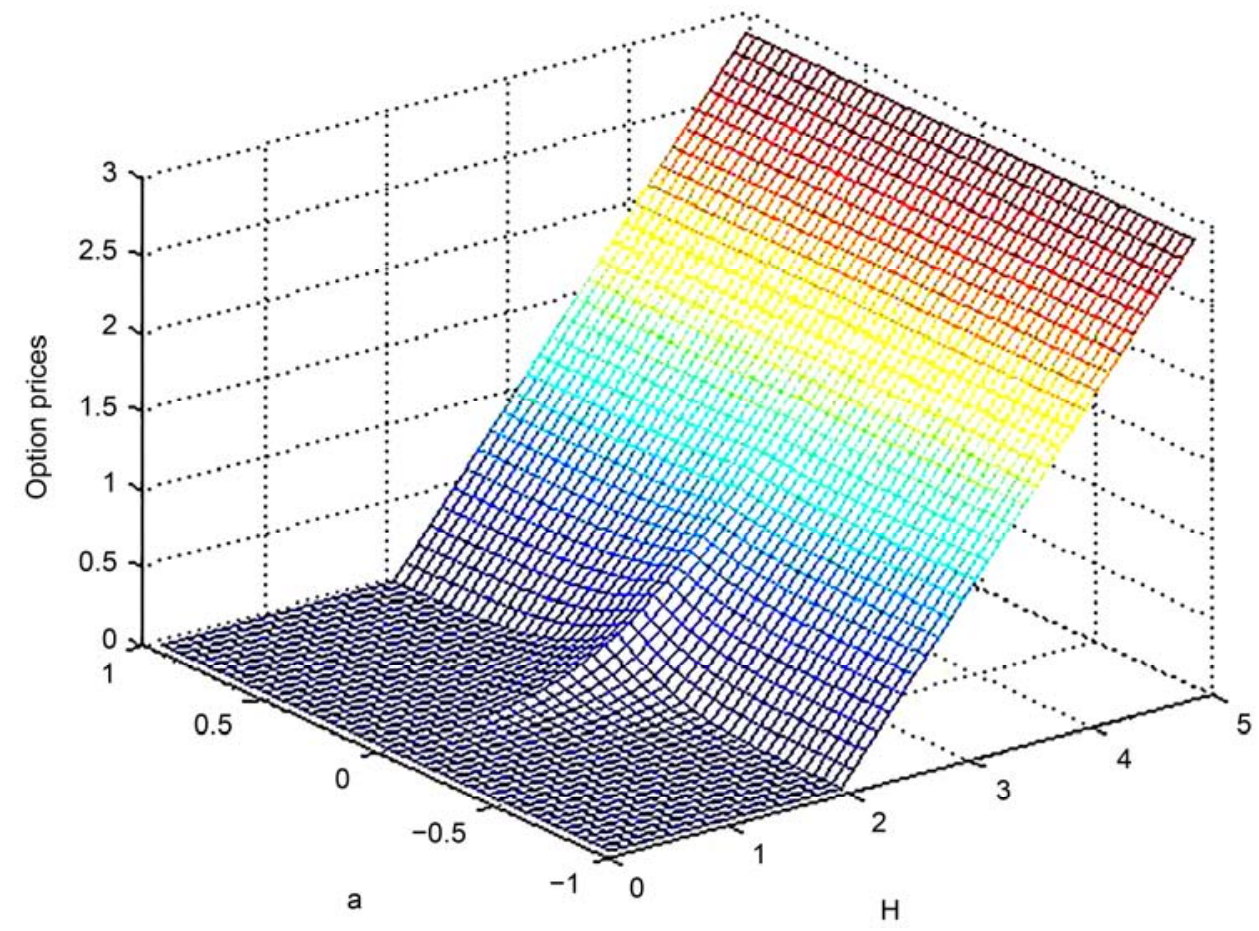

Figure 4. Prices for European options under constant correlation.

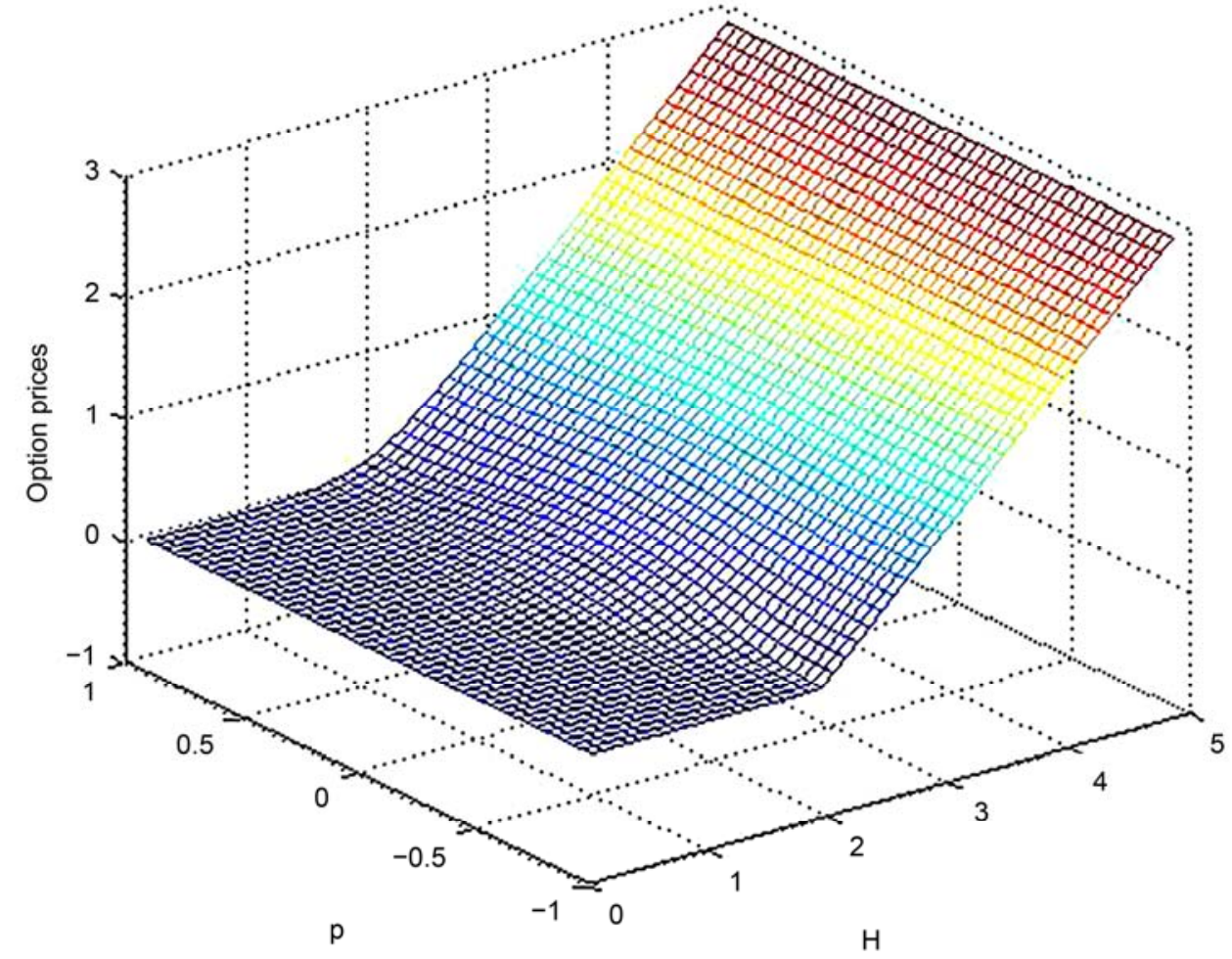

Figure 5. Prices for European options under stochastic correlation. 


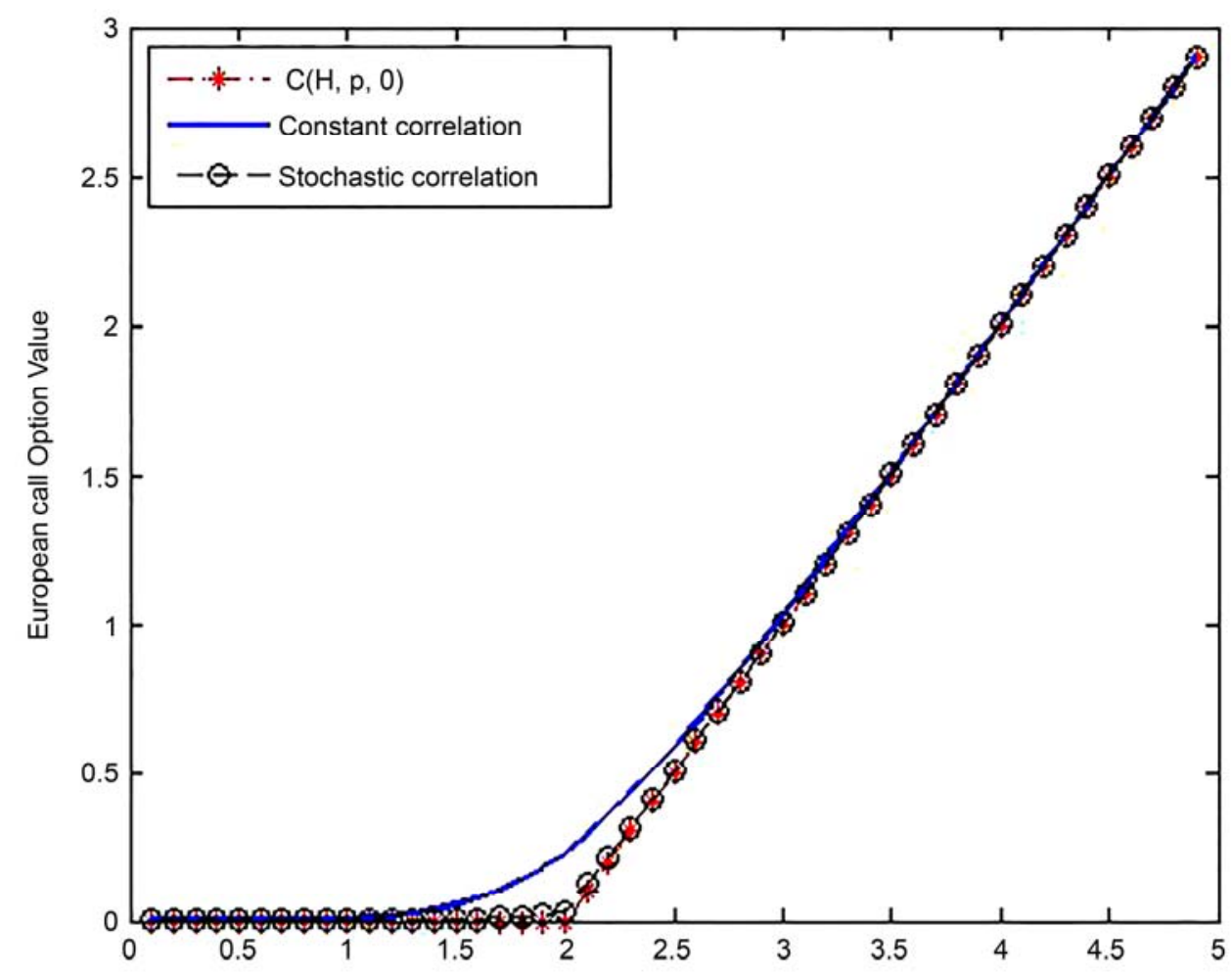

Figure 6. Values of European call option.

to be the domestic currency and South Africa to be the foreign currency. The data was first tested statistically and graphically before it was used. It was found that returns were heavily tailed and had no serial correlation, which implied that there was no direct dependency which could be exploited to predict tomorrows' returns based on today or previous day. Pricing equation for the European call with stochastic correlation performs better than that with constant correlation because its graph is very close to the graph of the market prices. Further work needs to be done in this area to improve the results such as considering volatility to be stochastic.

\section{Acknowledgements}

We thank African Union for financing this research.

\section{References}

[1] Merton, R.C. (1973) Theory of Rational Option Pricing. The Bell Journal of Economics and Management Science, 4, 141-183. http://dx.doi.org/10.2307/3003143

[2] Samuelson, P.A. (1965) Proof that Properly Anticipated Prices Fluctuate Randomly. Industrial Management Review, 6, 41-49.

[3] Black, F. and Scholes, M. (1973) The Pricing of Options and Corporate Liabilities. The Journal of Political Economy, 81, 637-654. http://dx.doi.org/10.1086/260062

[4] Cox, J.C., Ingersoll, J., Jonathan, E. and Ross, S.A. (1985) The Pricing of Options and Corporate Liabilities. Econometrica, 53, 363-384. http://dx.doi.org/10.2307/1911241

[5] van Emmerich, C. (2006) Modelling Correlation as a Stochastic Process. Preprints, 6.

[6] van Emmerich, C. (2016) Modelling Stochastic Correlation. Journal of Mathematics in In- 
dustry, 6, 1-18.

[7] Alvarez, A., Escobar, M. and Olivares, P. (2011) Pricing Two Dimensional Derivatives under Stochastic Correlation. International Journal of Financial Markets and Derivatives, 6, 265-287. http://dx.doi.org/10.1504/IJFMD.2011.045598

[8] Ma, J. (2009) Pricing Foreign Equity Options with Stochastic Correlation and Volatility. Annals of Economics and Finance, 10, 303-327.

[9] Grabbe, J.O. (1983) The Pricing of Call and Put Options on Foreign Exchange. Journal of International Money and Finance, 2, 239-253. http://dx.doi.org/10.1016/S0261-5606(83)80002-3

[10] Teng, L., Ehrhardt, M. and Günther, M. (2014) The Dynamic Correlation Model and Its Application to the Heston Model. Preprints.

[11] Teng, L., Ehrhardt, M. and Günther, M. (2015) The Pricing of Quanto Options under Dynamic Correlation. Journal of Computational and Applied Mathematics, 275, 304-310. http://dx.doi.org/10.1016/j.cam.2014.07.017 